\title{
FEBRE HEMORRÁGICA DA DENGUE: ASPECTOS EPIDEMIOLÓGICOS E ECONÔMICOS NO BRASIL
}

\author{
Dengue Hemorrhagic Fever: Epidemiological and Economic Aspects In Brazil
}

\author{
Aline Camargo de Oliveira ${ }^{1}$ \\ Ana Laura Oliveira Santos Dias Guimarães ${ }^{2}$ \\ Gabriela Lopes Antunes ${ }^{3}$ \\ Larissa Maria Almeida Ramos ${ }^{4}$ \\ Maria Cecília Drumond Cruz de Sales ${ }^{5}$ \\ Melanie Monteiro Rodrigues ${ }^{6}$ \\ Karina Andrade de Prince ${ }^{7}$
}

Resumo: Objetivo: Analisar o perfil clínico, epidemiológico e econômico da febre hemorrágica da dengue no Brasil. Método: Trata-se de um estudo, retrospectivo, descritivo, quantitativo, de base documental. Teve como universo de pesquisa a base de dados do Sistema de Informações Hospitalares do Sistema Único de Saúde, referente as internações, no período de 2011 a 2020. Resultados: No período avaliado foram registrados um total de 19.852 internações e 1.047 óbitos $(5,27 \%)$ por febre hemorrágica da dengue no país. A região Sudeste $(33,2 \%)$ e Nordeste $(30,9 \%)$, apresentaram maiores internações e, a maior taxa de mortalidade ocorreu na região Sul $(8,6 \%)$. Houve predomínio no sexo feminino $(52,26 \%)$, na faixa etária $10-19$ anos $(18,55 \%)$ e na cor/raça parda $(42,75 \%)$. A respeito do regime e caráter das internações, $34,44 \%$ foram em hospitais públicos e $95,8 \%$ por urgência. A

\footnotetext{
${ }^{1}$ Graduanda de Medicina pelo Centro Universitário FIPMoc - (UNIFIPMoc). Montes Claros/MG-Brasil. $\square$ line.camargo@ hotmail.com. https://orcid.org/0000-0002-8216-4070.

${ }^{2}$ Graduanda de Medicina pelo Centro Universitário FIPMoc - (UNIFIPMoc). Montes Claros/MG-Brasil. $\square$ analaurag_@hotmail.com. https://orcid.org/0000-0001-9661-7387.

${ }^{3}$ Graduanda de Medicina pelo Centro Universitário FIPMoc - (UNIFIPMoc). Montes Claros/MG-Brasil. $\square$ gabrielalopesantunes@gmail.com. https://orcid.org/0000-0002-6866-7931.

${ }^{4}$ Graduanda de Medicina pelo Centro Universitário FIPMoc - (UNIFIPMoc). Montes Claros/MG-Brasil. lariis3@hotmail.com. @ https://orcid.org/0000-0002-0595-786X.

${ }^{5}$ Graduanda de Medicina pelo Centro Universitário FIPMoc - (UNIFIPMoc). Montes Claros/MG-Brasil.

Maria.sales@aluno.unifipmoc.edu.br. https://orcid.org/0000-0003-3391-4805.

${ }^{6}$ Graduanda de Medicina pelo Centro Universitário FIPMoc - (UNIFIPMoc). Montes Claros/MG-Brasil. $\square$ melanie_monteiro@yahoo.com. 1 https://orcid.org/0000-0002-2617-0302.

${ }^{7}$ Doutora em Biociências e Biotecnologia Aplicadas a Farmácia (UNESP). Professora do Centro Universitário FIPMoc - (UNIFIPMoc). Minas Gerais. Brasil. $\square$ karina.prince@professor.unifipmoc.edu.br. https://orcid.org/0000-0001- 8231-852X.
}

$\begin{array}{ccc}\text { Recebido em } & \text { Aceito em } & \text { Publicado em } \\ 14 / 07 / 2021 & 26 / 10 / 2021 & 09 / 11 / 2021\end{array}$


maioria dos óbitos ocorreu entre pacientes do sexo masculino $(52,24 \%)$ e na faixa etária acima dos 70 anos $(17,48 \%)$. O valor total gasto com as internações foi de $11.936 .607,81$ reais, dos quais $34,44 \%$ foram destinados às internações do sistema público. Conclusão: As internações em decorrência dessa doença se encontram elevadas no Brasil, predominante em mulheres, jovens e pardas, proporcionando altos custos para a saúde pública do país.

Palavras-chave: Hospitalizações; Febre Hemorrágica da Dengue; Gastos em Saúde.

Abstract: Objective: To analyze the clinical, epidemiological and economic profile of dengue hemorrhagic fever in Brazil. Method: This is a retrospective, descriptive, quantitative, documentbased study. The universe of research was the database of the Hospital Information System of the Unified Health System, referring to admissions, in the period from 2011 to 2020. Results: In the period evaluated, a total of 19,852 admissions and 1,047 deaths were recorded $(5,27 \%)$ due to dengue hemorrhagic fever in the country. The Southeast $(33.2 \%)$ and Northeast $(30.9 \%)$ regions had higher hospitalizations and the highest mortality rate occurred in the South region $(8.6 \%)$. There was a predominance of females (52.26\%), aged 10-19 years (18.55\%) and mixed races (42.75\%). Regarding the regime and nature of admissions, $34.44 \%$ were in public hospitals and $95.8 \%$ were urgent. Most deaths occurred among male patients (52.24\%) and aged over 70 years (17.48\%). The total amount spent on hospitalizations was BRL 11,936,607.81, of which $34.44 \%$ were allocated to hospitalizations in the public system. Conclusion: Hospitalizations due to this disease are high in Brazil, predominantly in women, young people and mixed race, resulting in high costs for public health in the country.

Key words: Hospitalization; Dengue Hemorrhagic Fever; Health expenditures.

\section{INTRODUÇÃO}

A dengue é caracterizada como uma doença febril aguda, dinâmica e sistêmica ${ }^{1}$, cujo espectro clínico pode variar de um quadro assintomático a uma série de síndromes com manifestações clínicas graves. ${ }^{2}$ É uma doença transmitida pelo mosquito Aedes aegypti, que pode apresentar os quatro sorotipos virais - DENV-1, DENV-2, DENV-3 e DENV-4. ${ }^{3}$ A febre hemorrágica da dengue é uma das possíveis formas de evolução da doença, sendo que infecções subsequentes com sorotipos distintos de DENVs estão associadas a maior risco da complicação. ${ }^{4}$

O extravasamento de plasma é a principal propriedade da patogênese das manifestações hemorrágicas na doença. Estudos evidenciam que a resposta imunológica exacerbada contra o vírus também atinge as células humanas. Nesse sentido, constata-se que há uma alteração momentânea da integridade e coagulação dos vasos sanguíneos, que podem ser mediados por mecanismos das respostas imunes inata e adaptativa. Além disso, a presença das proteínas não estruturais, como NS2A, NS2B, NS4B e NS5 também promove 
uma função importante na patogenia da doença, levando à produção de quimiocinas e mediadores pró-inflamatórios. ${ }^{5,6}$

Estudos demonstram que indivíduos nos extremos de idade, obesos e portadores de comorbidades são mais propensos a ter quadros graves. Outros fatores como sorotipo do vírus e suscetibilidade genética individual também possuem influência no desenvolvimento de complicações. $^{7}$

A febre hemorrágica da dengue normalmente se inicia de maneira semelhante ao quadro clínico da dengue clássica ${ }^{8}$, tendo como sinais de alerta dores abdominais fortes e contínuas, vômitos persistentes, pele pálida, fria e úmida, manchas vermelhas na pele, sangramento de mucosa, sonolência, agitação, confusão mental, perda da consciência, sede excessiva, boca seca e pulso rápido e fraco. ${ }^{9}$

O diagnóstico da dengue é realizado com base em dados clínicos, epidemiológicos e laboratoriais, empregando-se, para este último, exames inespecíficos, como hemograma e prova do laço. ${ }^{10}$ Já os exames específicos estão direcionados para isolamento viral ou para pesquisa de anticorpos. Entre eles, o método mais utilizado é a sorologia. ${ }^{8}$

O aumento de hematócrito e a diminuição de plaquetas são indicadores relevantes do agravamento do quadro e do início da febre hemorrágica da dengue, sendo necessária a hospitalização imediata do indivíduo. A conduta para pacientes com dengue hemorrágica envolve a terapia com fluidos intravenosos, além do monitoramento clínico do hematócrito, contagem de plaquetas, pulsação, pressão arterial, temperatura, débito urinário e fluido administrado. ${ }^{2,11}$

O quadro pode se agravar rapidamente com grande diminuição de volume plasmático intravascular e consequente choque hipovolêmico, caracterizando a síndrome do choque da dengue, que pode levar à morte ${ }^{8}$. Para evitar este quadro, é recomendado o início precoce da reposição de fluidos e restabelecimento do volume correto. ${ }^{12}$

Tendo em vista que a transmissão da dengue se dá pelo mosquito Aedes aegypthi $^{13}$, as medidas profiláticas incluem a prevenção da picada de mosquitos ${ }^{14} \mathrm{e}$ a aplicação de inseticidas sintéticos ou naturais. ${ }^{15}$ No Brasil, como a dengue possui caráter sazonal, a vigilância epidemiológica representa, também, uma das estratégias mais eficientes para controlar os focos de mosquito. ${ }^{16}$ 
Outra medida a ser considerada envolve a recomendação da não utilização do ácido acetilsalicílico, uma vez que tal medicamento possui efeito anticoagulante e, consequentemente, é capaz de aumentar o risco de sangramento. Assim, evitar o uso do remédio pode amenizar, dentre outros aspectos, um possível quadro de trombocitopenia grave. ${ }^{17,18}$

Considerando o impacto da dengue na saúde pública, o governo tem aplicado políticas públicas de combate à dengue, como o Programa Nacional de Controle da Dengue, responsável por promover mudanças na forma de controle da doença, com foco na participação ativa da população. Dessa forma, a educação em saúde ganha ênfase, pois somente através da difusão de conhecimentos é que as mudanças de hábitos e comportamentos serão consolidadas. ${ }^{19}$

O grande número de internações hospitalares e os gastos relacionados com os indivíduos com febre hemorrágica da dengue fazem com que a doença se torne um problema de saúde pública. Assim, o objetivo do presente artigo foi analisar o perfil clínico, epidemiológico e econômico da febre hemorrágica devido ao vírus da dengue no Brasil.

\section{MÉTODOS}

Trata-se de um estudo retrospectivo, descritivo, quantitativo, de base documental com procedimento comparativo-estatístico. Teve como universo de pesquisa a base de dados do Sistema de Informações Hospitalares do Sistema Único de Saúde (SIH/SUS), referente ao perfil epidemiológico, clínico e econômico da febre hemorrágica da dengue, de acordo com as regiões do Brasil, no período de 2011 a dezembro de 2020. Os dados foram obtidos a partir do SIH/SUS, disponibilizados pelo Departamento de informática do Sistema Único de Saúde (DATASUS), no endereço eletrônico (http://www2.datasus.gov.br).

A coleta de dados ocorreu nos meses de fevereiro e março de 2021 por meio da utilização do programa de Informações em Saúde (TABNET). A tabulação dos registros do Sistema de Informações Hospitalares do Sistema Único de Saúde (SIH/SUS) para a pesquisa incluiu as variáveis sociodemográficas (sexo, faixa etária e etnia) e clínicas (número de internações e média de permanência, óbitos, caráter, regime de atendimento, bem como os custos das internações), reconhecendo a limitação do estudo, devido ao acesso a dados secundários. 
Os dados populacionais para cálculo da prevalência média de casos de internações por febre hemorrágica da dengue de acordo com as regiões do Brasil foram obtidos a partir do portal do Instituto Brasileiro de Geografia e Estatística (IBGE, 2020). Foi realizada análise descritiva das variáveis, com frequência, porcentagem e a média do número de casos registrados. A análise bivariada foi realizada por meio do teste Qui-quadrado de Pearson.

Foi considerado como nível de significância o valor de $\mathrm{p} \leq 0,05$. Para análise estatística (análise descritiva e teste qui-quadrado) foi utilizado o software Excel 12.0 (Office 2007) e o programa Statistical Package for the Social Sciences (SPSS) para Windows, versão 23.0 e Origin 7.1

Tendo em vista que a pesquisa se baseou em dados disponibilizados em meio eletrônico pelo Ministério da Saúde, sendo esses de domínio público e, pelo fato de haver sigilo acerca das informações de identificação inerentes aos seres humanos envolvidos, esse estudo dispensa a apreciação e a aprovação pelo Comitê de Ética em Pesquisa.

\section{RESULTADOS}

No período de 2011 a 2020 foram registrados um total de 19.852 internações por febre hemorrágica da dengue no Brasil. O número variou de 721 a 4.206, com uma média de 1.985 casos por ano. Observou-se diminuição do número de internações entre 2011 - 2017 (82,8\%) e 2019 - 2020 (56,8\%), um aumento entre 2017 e 2019 (443,8\%), oscilações entre 2012 e 2016 e picos nos anos de 2011, 2015 e 2019 (Figura 1).

Figura 1: Número de internações por febre hemorrágica da dengue no Brasil, 2011 a 2020.

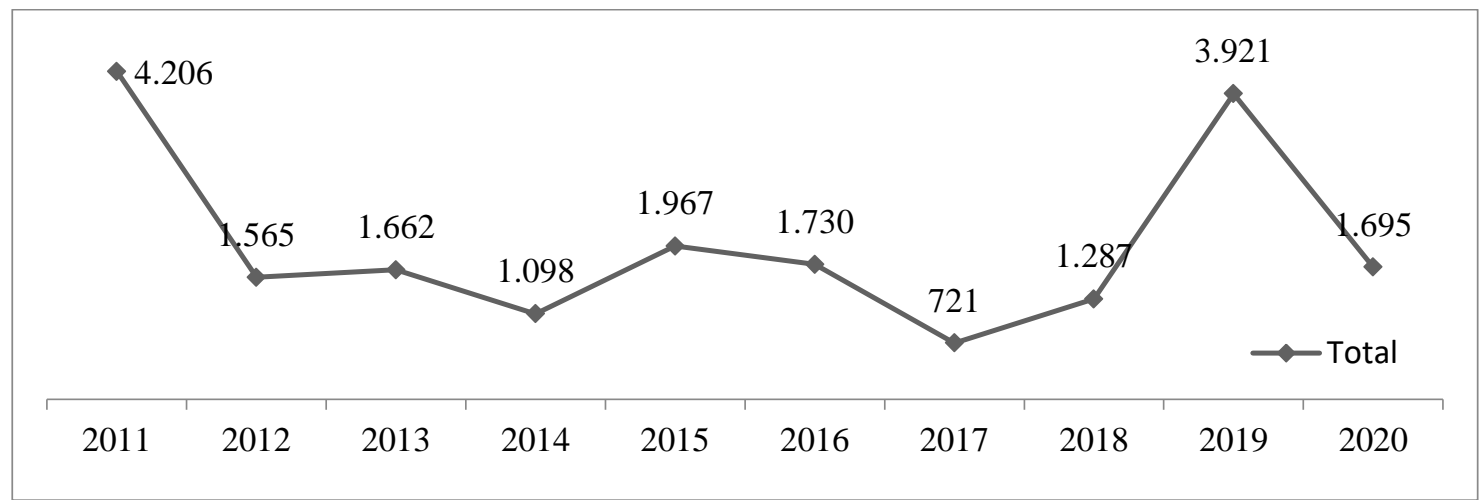

Fonte: Ministério da Saúde - Sistema de Informações Hospitalares do SUS (SIH/SUS).

Em relação à distribuição das internações por febre hemorrágica da dengue de acordo com as regiões do Brasil, observa-se um maior número de notificações nas regiões 
Sudeste $(6.592 / 33,2 \%)$ e Nordeste $(6.154 / 30,9 \%)$. Quanto à taxa de mortalidade, a maior encontra-se na região $\operatorname{Sul}(8,6 \%)$ e a menor taxa encontra-se na região Centro-Oeste $(4,08 \%)$ (Figura 2).

Figura 2: Número de internações e taxa de mortalidade por febre hemorrágica da dengue no Brasil, 2011 a 2020.Fonte: Ministério da Saúde - Sistema de Informações Hospitalares do SUS (SIH/SUS).

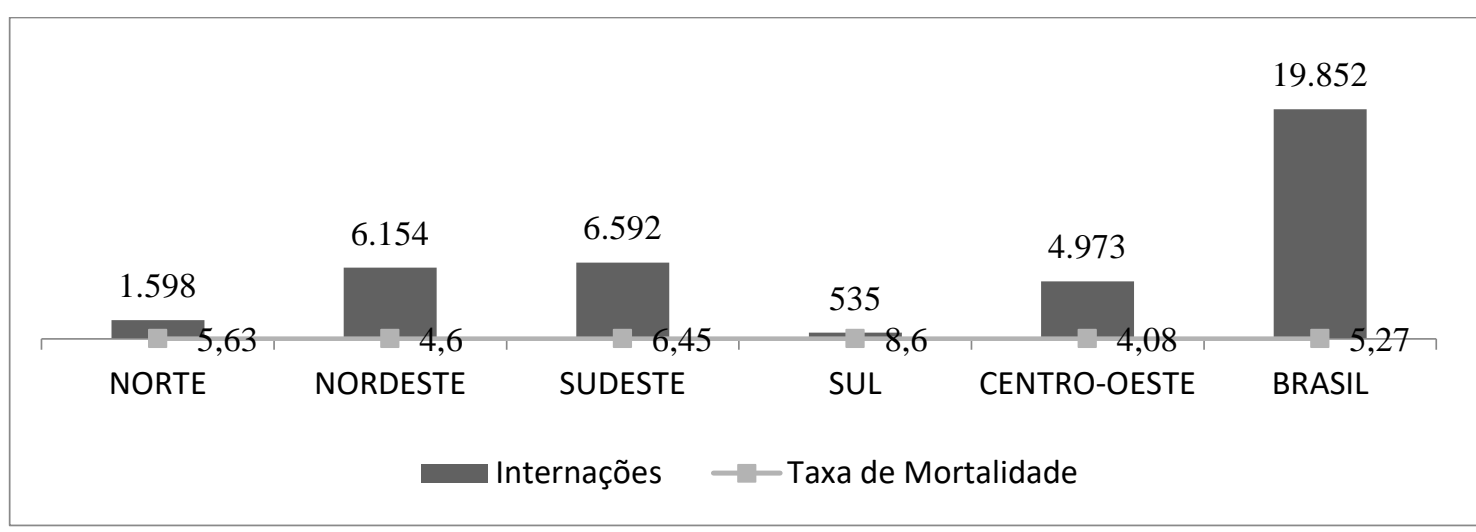

Analisando a prevalência média de internações por febre hemorrágica da dengue, entre os anos de 2011 e 2020, observa-se uma maior prevalência na região Centro-Oeste (30,1 casos/100 mil habitantes) e menor prevalência na região Sul (1,8 casos/100 mil habitantes) (Figura 3).

Figura 3: Mapa epidemiológico da prevalência da febre hemorrágica da dengue, de acordo com as regiões brasileiras, 2011 a 2020.

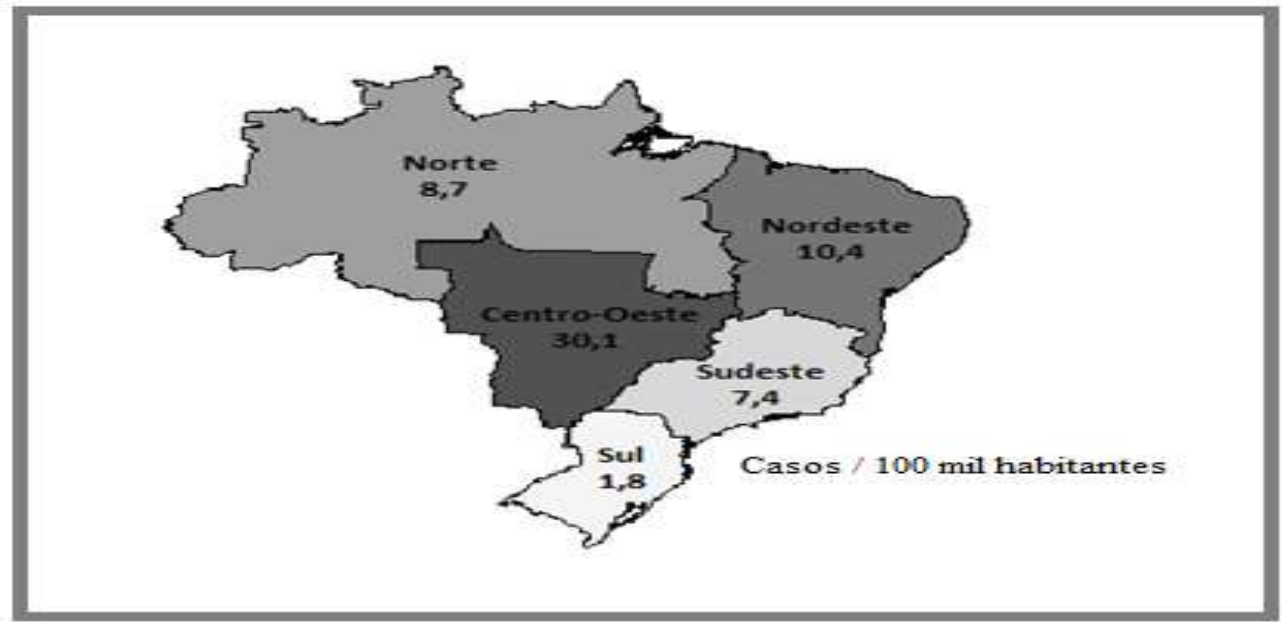

Fonte: Ministério da Saúde - Sistema de Informações Hospitalares do SUS (SIH/SUS).

De acordo com os dados sociodemográficos e clínicos dos pacientes analisados, houve diferenças estatisticamente significativas entre essas variáveis $(\mathrm{p}<0,001)$. Nota-se o 
predomínio da doença no sexo feminino $(52,26 \%)$, na faixa etária $10-19$ anos $(18,55 \%)$ e na cor/raça parda (42,75\%). Quanto ao caráter de atendimento, a maior parte dos casos foram de urgência $(95,8 \%)$. Houve um predomínio no regime público $(34,44 \%)$ durante as internações, sendo que a média de permanência neste regime foi de 5,1 dias, apresentando-se maior que no sistema privado (Tabela 1).

No que se refere aos gastos públicos, o valor total foi de $11.936 .607,81$ reais, dos quais $34,44 \%$ foram destinados às internações do sistema público e 50,36\% encontravam-se sem informação (Tabela 1). O valor médio das internações no período foi de cerca de 535, 57 reais por paciente.

Em relação aos dados sociodemográficos e clínicos relativos aos óbitos dos pacientes internados por febre hemorrágica da dengue, houve diferenças estatisticamente significativas entre as variáveis avaliadas ( $\mathrm{p}<0,001)$. Dentre o total de 1.047 óbitos ocorridos no período analisado, foi observado um maior número de mortes entre os pacientes do sexo masculino $(52,24 \%)$, com idade igual ou superior a 70 anos $(17,48 \%)$, na cor/raça parda $(37,72 \%)$, internados em regime público $(35,91 \%)$, em caráter de urgência (98\%) (Tabela 2).

Tabela 1: Dados sociodemográficos e clínicos de pacientes internados por febre hemorrágica da dengue no Brasil, 2011 a 2020.

\begin{tabular}{llll}
\hline Variáveis & Total & $\%$ & Valor de $p$ \\
\hline Total & 19.852 & 100 & $<0,001$ \\
\hline Sexo & & & \\
\hline Masculino & 9.478 & 47,74 & $<0,001$ \\
Feminino & 10.374 & 52,26 & \\
\hline Faixa etária & & & \\
\hline Menor que 10 anos & 3.080 & 15,50 & \\
10 a 19 anos & 3.672 & 18,55 & \\
20 a 29 anos & 3.145 & 15,85 & \\
30 a 39 anos & 2.915 & 14,70 & \\
40 a 49 anos & 2.450 & 12,30 & \\
50 a 59 anos & 2.019 & 10,20 & \\
60 a 69 anos & 1.412 & 7,10 & \\
$\geq 70$ anos & 1.159 & 5,80 & \\
\hline Cor/raça & 4.262 & 21,61 & \\
\hline Branca & & & \\
\hline
\end{tabular}




\begin{tabular}{|c|c|c|c|}
\hline Preta & 375 & 3,38 & \\
\hline Parda & 8.074 & 42,75 & \\
\hline Amarela & 574 & 1,29 & \\
\hline Indígena & 26 & 0,47 & \\
\hline Sem informação & 3.540 & 30,47 & \\
\hline Regime & & & $<0,001$ \\
\hline Público & $4.111 .730,08$ & 34,44 & \\
\hline Privado & $1.813 .391,065$ & 15,20 & \\
\hline Sem informação & $6.011 .456,08$ & 50,36 & \\
\hline Caráter & & & $<0,001$ \\
\hline$\overline{\text { Eletivo }}$ & 831 & 4,2 & \\
\hline Urgência & 19.021 & 95,8 & \\
\hline Média de permanência & & & Não se aplica \\
\hline Público & 5,1 & & \\
\hline Privado & 4,4 & & \\
\hline Sem informação & 4,4 & & \\
\hline Total & 4,8 & & \\
\hline \multicolumn{4}{|l|}{ Valor Total } \\
\hline Público & $4.111 .730,08$ & 34,44 & \\
\hline Privado & $1.813 .391,65$ & 15,20 & \\
\hline Sem informação & $6.011 .486,08$ & 50,36 & \\
\hline Total & $11.936 .607,81$ & 100 & \\
\hline
\end{tabular}

Fonte: Ministério da Saúde - Sistema de Informações Hospitalares do SUS (SIH/SUS)

Tabela 2: Dados sociodemográficos e clínicos dos óbitos por febre hemorrágica da dengue no Brasil, 2011 a 2020.

\begin{tabular}{llll}
\hline Variáveis & Total & $\%$ & Valor de $p$ \\
\hline Total & 1.047 & 100 & \\
\hline Sexo & & & $<0,001$ \\
\hline Masculino & 547 & 52,24 & \\
Feminino & 500 & 47,76 & \\
\hline Faixa etária & & & \\
\hline Menor que 10 anos & 84 & 8,02 & \\
10 a 19 anos & 98 & 9,36 & \\
20 a 29 anos & 116 & 11,07 & \\
30 a 39 anos & 147 & 14,04 &
\end{tabular}




\begin{tabular}{|c|c|c|c|}
\hline 40 a 49 anos & 140 & 13,38 & \\
\hline 50 a 59 anos & 156 & 14,90 & \\
\hline 60 a 69 anos & 123 & 11,75 & \\
\hline$\geq 70$ anos & 183 & 17,48 & \\
\hline Cor/raça & & & $<0,001$ \\
\hline Branca & 234 & 22,35 & \\
\hline Preta & 26 & 2,48 & \\
\hline Parda & 395 & 37,72 & \\
\hline Amarela & 23 & 2,20 & \\
\hline Indígena & 04 & 0,39 & \\
\hline Sem informação & 365 & 34,86 & \\
\hline Regime & & & $<0,001$ \\
\hline Público & 376 & 35,91 & \\
\hline Privado & 170 & 16,23 & \\
\hline Sem informação & 501 & 47,86 & \\
\hline Caráter & & & $<0,001$ \\
\hline Eletivo & 21 & 2,00 & \\
\hline Urgência & 1026 & 98,00 & \\
\hline
\end{tabular}

Fonte: Ministério da Saúde - Sistema de Informações Hospitalares do SUS (SIH/SUS)

\section{DISCUSSÃO}

No período de 2011 a 2020, foram registrados um total de 19.582 casos de internações por febre hemorrágica da dengue (FDH) no Brasil. Observou-se um aumento no número de internações entre 2017 e 2019 (443,8\%) e um decréscimo mais acentuado entre 2011 e 2017 (82,8\%) e entre 2019-2020 (56,8\%), mantendo-se uma pequena variação nos outros anos. Tal fato pode ser explicado por diversos fatores, como a implantação da Estratégia Global para Prevenção e Controle da Dengue pela OMS, no ano de 2012, que visava uma diminuição da mortalidade em $50 \%$ e da morbidade em $25 \%$ até $2020 .{ }^{20}$

Outras medidas podem ter justificado a redução desse número. As ações de educação populacional, com o intuito de informar e mobilizar os cidadãos, têm extrema importância, pois atingem grandes massas, através de campanhas ou por meio da atuação dos agentes de saúde em cada moradia. As estratégias de controle biológico, por sua vez, são direcionadas ao vetor, e consistem em aplicação de larvicidas, uso de fumacê e utilização de organismos vivos capazes de eliminar o vetor. Assim, os cuidados individuais e coletivos 
contribuem para diminuir os criadouros do agente transmissor da dengue e, consequentemente, impactam na redução de novos casos dessa doença. ${ }^{21}$

Entretanto, percebe-se que os objetivos dos programas até hoje apresentados no Brasil para o controle da dengue não foram totalmente atingidos, uma vez que os anos de 2011, 2015 e 2019 foram críticos quando comparados aos outros anos. Fatores como variação climática significativa, eventos de ordem ambiental, socioeconômicos ou de comportamento populacional que tenham ocorrido neste período podem ter influenciado a ocorrência desses picos. Além disso, o crescimento populacional urbano aliado ao número de sorotipos da dengue, a falhas de prevenção e de controle da doença e do mosquito Aedes aegypti corroboram para o aumento dos números de casos. ${ }^{22}$

Em relação à taxa de mortalidade, a região Sul ocupou a primeira posição $(8,6 \%)$, seguida pela região Sudeste $(6,45 \%)$. Estas regiões possuem alta taxa de mortalidade devido às alterações climáticas, elevadas taxas de pluviosidade e uma desordem no planejamento urbano. Assim, os casos de dengue hemorrágica são, por consequência, mais evidentes e mais frequentes nessas regiões. ${ }^{23}$

A dificuldade de acesso, falta de ações e capacitações de profissionais de saúde voltadas especificamente para os riscos da dengue hemorrágica, bem como a baixa qualidade do atendimento são importantes definidores do número elevado na taxa de mortalidade. ${ }^{24}$ Entretanto, apesar de serem regiões que apresentam maior desenvolvimento econômico e disponibilidade de serviços de saúde, as regiões Sul e Sudeste expressam elevadas taxas de mortalidade.

Quanto aos aspectos sociodemográficos, os resultados encontrados revelaram um número maior de internações entre indivíduos do sexo feminino $(52,26 \%)$, na faixa etária 1019 anos $(18,55 \%)$ e da cor/raça parda (42,75\%), havendo diferenças estatisticamente significativas entre as variáveis $(\mathrm{p}<0,001)$. Esses dados se assemelham aos encontrados em outros estudos realizados no país. Os achados em relação à etnia podem estar associados ao predomínio da cor/raça parda no Brasil, de acordo com o IBGE. ${ }^{25,26,27}$

Quanto ao número de internações segundo o regime, 34,44\% ocorreram em hospitais públicos, representando a maioria entre os regimes não-ignorados. Estes resultados podem estar associados à fragilidade por parte do serviço público em indicadores 
socioespaciais, como o saneamento básico, que levam ao desenvolvimento de regiões vulneráveis ao risco de dengue. ${ }^{28}$

Além disso, a média do tempo de permanência dos pacientes no setor público foi de 5,1 dias e no setor privado de 4,4 dias, demandando maiores gastos para o sistema de saúde público, já que a febre hemorrágica da dengue consiste na forma mais crítica da doença, necessitando, assim, de mais intervenções. ${ }^{29}$

No que tange aos gastos, o valor total foi de $11.936 .607,81$ reais e o custo médio das internações cerca de 535, 27 reais. Houve um considerável custo, principalmente no sistema de saúde público, totalizando $34,44 \%$ dos valores registrados, comparado a um total de 15,20\% no sistema de saúde privado, havendo uma diferença de 19,24\%. Essa disparidade pode ser explicada devido ao maior número de internações ocorrerem no setor público, pois, segundo dados do regime, o sistema público obteve $34,44 \%$ das internações, além de uma maior média de permanência em relação ao setor privado.

No que diz respeito ao elevado número de internações em caráter de urgência, que correspondeu à 95,8\%, pode-se deduzir que aspectos como dificuldades no incentivo às pesquisas e um serviço público ainda pouco efetivo em fornecer um diagnóstico em tempo adequado e preciso sejam fatores que possam contribuir para a situação apresentada. ${ }^{30}$

Percebe-se, por exemplo, que a maioria dos eventos fatais da dengue está relacionada à identificação tardia da doença, favorecendo o agravamento dos distúrbios hemodinâmicos. Um dos fatores determinantes para dificultar a evolução do quadro é a capacidade dos médicos de identificar os fatores de risco para choque, fenômeno responsável pela maioria dos óbitos em pacientes com febre hemorrágica da dengue. Outros fatores como sorotipo do vírus, suscetibilidade genética individual e intensidade do extravasamento plasmático também possuem influência no desenvolvimento de complicações ${ }^{7}$.

Em relação aos óbitos dos pacientes internados por febre hemorrágica da dengue, dentre o total de $1.047(5,27 \%)$ óbitos ocorridos no período analisado, foi observado um maior número entre os pacientes do sexo masculino (52,24\%), com idade igual ou superior a 70 anos $(17,48 \%)$, na cor/raça parda $(37,72 \%)$, sendo que na população economicamente ativa os óbitos chegaram a 53,4\%. O estudo realizado por Pontes e colaboradores também ratifica esse resultado, onde 53,23\% dos pacientes que evoluíram a óbito eram do sexo masculino, $19,14 \%$ com idade acima de 70 anos e $46,7 \%$ na faixa etária economicamente ativa ${ }^{26}$. 
O considerável número de óbitos resulta em um forte impacto socioeconômico para o Brasil, devido aos elevados gastos com internação e potenciais anos de vida perdidos, já que a maior parte dos óbitos ocorrem na população economicamente ativa. ${ }^{31} \mathrm{O}$ custo médio de internação hospitalar pela doença chega a $\mathrm{R} \$ 527,00$ por paciente ${ }^{29}$, valor também destacado no presente estudo.

Diante da permanência do alto número de casos de dengue hemorrágica no país e da eficácia parcial de estratégias de controle epidemiológico da doença, é necessário identificar as falhas das políticas de intervenção, visando à redução do número de internações, óbitos e gastos hospitalares, além de proporcionar à saúde pública caminhos concisos e financeiramente mais estáveis. ${ }^{32}$

Vale ressaltar que estudos recentes demonstram que intervenções individuais parecem ser mais viáveis financeiramente. Entretanto, o impacto epidemiológico é maior quando as intervenções são combinadas, incluindo tanto medidas como vacinação, como rotação de inseticidas, mosaico (alternância entre dois produtos com diferentes modos de ação) ou mistura de inseticidas (uso simultâneo dos agrotóxicos). ${ }^{33}$ Assim, a perspectiva relacionada ao controle da doença perpassa pelo trabalho concomitante dos agentes de saúde, bem como pela consciência individual, visando à potencialização do combate ao vetor, através de medidas como educação em saúde, mobilização da comunidade, identificação dos criadouros, entre outros. ${ }^{34}$

Em relação à vacinação, por ser um país predominantemente de clima tropical e, consequentemente, apresentar maior circulação do vetor, observou-se a necessidade de direcionar ações e estratégias com o objetivo de prevenção da dengue, levando à criação de uma vacina eficaz e segura contra os quatro sorotipos. A tetravalente Dengvaxia é a única vacina atualmente licenciada no país, apresentando uma eficácia média de $60 \%{ }^{35}$

Estudos demonstram que o uso da vacina em indivíduos com infecção prévia pode reduzir a chance de casos graves de dengue, bem como o número de internações hospitalares. ${ }^{36}$ Entretanto, parece não funcionar de forma eficaz em indivíduos menores de nove anos, além de poder levar à dengue grave em casos de infecção secundária com um sorotipo diferente do vírus, sendo, por isso, proibida em alguns países. ${ }^{37}$

A partir do crescimento expressivo de internações observado no ano de 2019, hipóteses são sugeridas pelos pesquisadores, como fenômenos climáticos, a exemplo do El 
Niño, influenciando diretamente na pluviosidade e temperatura, favorecendo, assim, a proliferação do vetor. Além disso, eventos de nível nacional, como eleições e Copa do Mundo, ocorridos em 2018, podem ter contribuído com o enfraquecimento das políticas públicas direcionadas ao combate ao vetor, justificando o aumento das internações no ano subsequente.

A limitação do estudo foi o uso de dados secundários, adquiridos na base de dados do DATASUS, visto que eles podem interferir na análise, sobretudo pela baixa completude dos registros. Por conseguinte, é indispensável garantir a sensibilidade e a confiabilidade dos dados, para a sua utilização com a devida segurança por pesquisadores, gestores e profissionais da saúde de igual forma no território nacional.

\section{CONSIDERAÇÕES FINAIS}

Conclui-se, assim, que o número de internações pela febre hemorrágica da dengue apresentou uma redução entre os anos de 2011-2017 e 2019-2020, mas aumentou expressivamente entre 2017 e 2019 . Houve acometimento predominante no sexo feminino, na faixa etária entre 10-19 anos e na cor/raça parda. Em relação ao caráter de atendimento, a maior parte dos casos foram de urgência e no sistema público, gerando gasto total de 11.936.607,81 reais.

Faz-se necessário, portanto, o aprimoramento de políticas públicas que visem ao controle vetorial, educação em saúde e diagnóstico e manejo precoces, medidas que teriam um impacto significativo no número de internações e óbitos pela febre hemorrágica da dengue. Consequentemente, haveria uma redução das complicações e gastos públicos com a doença.

\section{REFERÊNCIAS}

1- BRASIL. Ministério da Saúde. Secretaria de Vigilância em Saúde. Dengue: diagnóstico e manejo clínico no adulto e na criança - 5a edição, Brasília, 2016. Disponível em: dengue-manejo-adulto-crianca-5d.pdf (saude.gov.br). Acesso em: 09 out. 2020.

2- KHETARPAL, Niyati, KHANNA Ira. Dengue fever: causes, complicationns, and vaccine strategies. Journal of immunology Research, London, v. 2016, p. 1-14, jul. 2016. 
3- HARAPAN, Harapan et al. Dengue: A minireview. Viruses, Lima, v. 12, n. 8, p. 829, jul, 2020.

4- WANG, Wen-Hung et al. Dengue hemorrhagic fever - A systemic literature review of current perspectives on pathogenesis, prevention and control. Journal of Microbiology, Immunology and Infection, Kaohsiung, v. 53, n. 6, p. 1-16, dez, 2020.

5- RAZA, Muhammad Ali et al. A case of Dengue Fever With Hemorrhagic Manifestations. Cureus, Palo Alto, v. 12, n. 6, p. 1-14, jun, 2020.

6- SRIKIATKHACHORN, Anon; MATHEW, Anuja; ROTHMAN, Alan L. Immunemediated cytokine storm and its role in severe dengue. Seminars in Immunopathology, Berlim, v. 39, n. 5, p. 563-574, abr, 2017.

7- NELWAN, Erni. J. Early Detection of Plasma Leakage in Dengue Hemorrhagic Fever. Acta Med Indones, Indonésia, v. 50, n. 3, p. 183-184, jul, 2018.

8- FERREIRA, Wellington Fernando da Silva; OLIVEIRA, Elia Machado de; DUTRA, Denecir de Almeida. Um breve panorama compreensivo sobre a dengue: reflexões clínicas e percepções comunitárias. Revista Jurídica Uniandrade, Curitiba, v. 1, n. 31, p. 6-7, jan-jul, 2020.

9- LUZ, Kleber Giovanni et al. Comparação da gravidade dos casos de dengue segundo a classificação antiga e a classificação revisada. Revista de Medicina, São Paulo, v. 6, n. 97, p. 549-551, nov-dez, 2018.

10-BIASSOTI, Amabile Visioti; ORTIZ, Mariana Aparecida Lopes. Diagnóstico laboratorial da dengue. Revista Uningá, Maringá, v. 29, n. 1, p. 123-124, mar, 2017.

11- LEE, Tau Hong et al. Current management of severe dengue infection. Expert Review of Anti-infective therapy, London, v. 15, n. 1, p. 67-78, out, 2016.

12-DONDORP, Arjen M.; HOANG, Mai Nguyen Thi Hoang; MER, Mervyn.. Management of Severe Malaria and Severe Dengue in Resource-Limited Settings. Intensive Care Medicine, Bethesda, v. 43, n. 11, p. 1692-1685, fev, 2019.

13-GUO, Congcon et al. Global epidemiology of dengue outbreaks in 1990-2015: a systematic review and meta-analysis. Frontiers in Cellular and Infection Microbiology, Lausanne Switzerland, v. 7, p. 317, jul, 2017. 
14- SCHAEFER, Timothy J.; PANDA, Prasan K.; WOLFORD, Robert W. Dengue Fever. StatPearls Publishing, Treasure Island, p. 1-18, ago, 2020.

15- JASAMAI, Malina et al. Current prevention and potential treatment options for dengue infection. Journal of Pharmacy \& Pharmaceutical Sciences, Alberta, v. 22, p. 440-456, set, 2019.

16-DOS SANTOS, Gabriel Augusto Cordeiro dos et al. Dengue: Prevenção, Controle e Cuidados de Enfermagem - Revisão Integrativa da Literatura 2008-2013. Revista Brasileira de Ciências da Saúde, Belém, v. 20, n. 1, p. 71-78, abr, 2016.

17- ORANTES, Luis Del Carpio. Dengue en poblaciones especiales. Revista del Hospital Juárez de México, Veracruz, v. 86, n. 1, p. 33-38, jan-mar, 2019.

18-DA SILVA, Edillaine Lemes et al. Prevenção da Dengue: Experiências Escolares. Perspectivas Experimentais e Clínicas, Inovações Biomédicas e Educação em Saúde (PECIBES), Campo Grande, v. 3, n. 2, p. 66-73, nov, 2017.

19- SILVA, Ivanise Brito; MALLMANN, Danielli Gavião; DE VASCONCELOS, Eliane Maria Ribeiro. Estratégias de combate à dengue através da educação em saúde: uma revisão integrativa. Revista Saúde (Santa Maria), Santa Maria, v. 41, n. 2, p. 27-34, jul-dez, 2015.

20-CORREIA, Tercio Cirqueira et al. Prevalência de dengue clássica e dengue hemorrágica no Brasil, entre 2011 e 2015. Revista Eletrônica Acervo Saúde, São Paulo, v. 22, n. 22, p.1-8, abr, 2019.

21-CANGIRANA, Jaqueline Ferreira; RODRIGUES, Gabriela Meira de Moura. Diferenças entre dengue clássica e hemorrágica e suas respectivas medidas profilática. Revista liberum Accessum, Brasília, v.1, n.1, p. 30-38, mar, 2020.

22- ARAÚJO, Amanda Bezerra de. Uma análise das políticas de controle e combate à dengue no Brasil. Dissertação de mestrado (Programa de Pós Graduação em Gestão e Economia da Saúde) - Universidade Federal de Pernambuco, Recife, 158 p. 2018.

23-RIBEIRO, Ana Clara Machado et al. Condições Socioambientais relacionadas à Permanência da Dengue no Brasil-2020. Revista Saúde e Meio Ambiente, Três Lagoas, v. 11, n. 2, p. 326-340, ago-dez, 2020.

24- MOTA, Anne Karin Madureira da et al. Mortalidade materna e incidência de dengue na Região Sudeste do Brasil: estudo ecológico no período 2001-2005. Cadernos de Saúde Pública, Rio de Janeiro, v. 28, p. 1057-1066, jun, 2012. 
25- DUTRA, Fabiana Caetano Martins Silva; COSTA, Letícia Cardoso; SAMPAIO, Rosana Ferreira. A influência do afastamento do trabalho na percepção de saúde e qualidade de vida de indivíduos adultos. Fisioterapia e Pesquisa, Uberaba, v. 23, n. 1, p. 98-104, jan-mar, 2016.

26- PONTES, Diego de Sousa et al. Morbimortalidade por febre hemorrágica da dengue no brasil. Temas em Saúde, João Pessoa, v. 20, n. 4, p. 193-205, ago, 2020.

27-GONÇALVES, Caio Willer Brito et al. Análise de aspectos epidemiológicos da dengue no estado do Tocantins. Revista de Patologia do Tocantins, Palmas, v. 6, n. 4, fev, 2020.

28- ALMEIDA, Rayane Brito de; FERREIRA, Marcela Beleza de. Especialização da dengue: indicadores socioespaciais e aspectos climáticos no processo saúdedoença. Raega - O Espaço Geográfico em Análise, Curitiba, v. 45, n. 1, p. 200-213, dez, 2018.

29- OLIVEIRA, Dayane de Lima et al. Custo das internações por dengue no estado de Goiás, no período de 2016 a 2018. Brazilian Journal of Development, Curitiba, v. 6, n. 5, p. 30.695-30.697, maio, 2020.

30-SANTOS, Maressa Martins; SANTOS, Rebeca Isis de Oliveira; UEHARA, Sílvia Carla da Silva André. Perfil epidemiológico da dengue: subsídios para os serviços de saúde. Revista Recien, São Paulo, v.10, n. 30, p.117-128, mar, 2020.

31-PIMENTEL, Thales Lemos et al. Anos potenciais de vida perdidos no brasil em decorrência da dengue: impacto socioeconômico. Health Residencies Journal, Viçosa, v. 1, n. 7, p. 1-11, set, 2020.

32- LASNEAUX, Marcello Vieira. Programas de controle da dengue no brasil: uma análise crítica dos seus principais objetivos. Revista eixo, Brasília, v.6, n.2, p.87-92, jul-dez, 2017.

33- KNERER, Gerhart; CURRIE, Christine S.M; BRAILSFORD, Sally C. The economic impact and cost-effectiveness of combined vector-control and dengue vaccination strategies in Thailand: Results from a dynamic transmission model. PLOS Neglected Tropical Diseases, San Francisco, Califórnia, EUA, v.14, n.10, oct, 2020.

34- ANDRADE, Juliana Nascimento; PEIXOTO, Thais Moreira; COELHO, Maíra Moreira Peixoto. Visita do agente de combate às endemias frente pandemia por covid19: desafios e perspectivas. Revista de Divulgação Científica Sena Aires, Valparaiso de Goiás - Goiás, v.9, n.4, p.709-716, out-dez, 2020. 
35- DOURADO, Jeane de Morais; ARAÚJO, Christian Souza de; GODÓI, Isabella Piassi Dias. Hospitalizações, óbitos e gastos com dengue entre 2000 e 2015 na região norte do Brasil. Revista Brasileira Interdisciplinar de Saúde, Brasília - Distrito Federal, v.2, n.4, p.32-39, set,2020.

36- NASAR, Sitara; RASHID, Naeem; IFTIKHAR, Saima. Dengue proteins with their role in pathogenesis, and strategies for developing an effective anti-dengue treatment: A review. Journal of medical virology, v.98, n.2, p. 941-955, aug, 2020.

37-FATIMA, Khunsha; SYED, Najah Irfan. Dengvaxia controversy: impact on vaccine hesitancy. Journal of Global Health, Edimburgo, Reino Unido, v.8, n. 2, oct, 2018. 